\title{
Noncontinuous Froude Number Scaling for the Closure Depth of a Cylindrical Cavity
}

\author{
Stephan Gekle, Arjan van der Bos, Raymond Bergmann, Devaraj van der Meer, and Detlef Lohse \\ Physics of Fluids Group and J. M. Burgers Centre for Fluid Dynamics, University of Twente, \\ P.O. Box 217, 7500AE Enschede, The Netherlands \\ (Received 18 December 2006; revised manuscript received 20 July 2007; published 27 February 2008)
}

A long, smooth cylinder is dragged through a water surface to create a cavity with an initially cylindrical shape. This surface void then collapses due to the hydrostatic pressure, leading to a rapid and axisymmetric pinch-off in a single point. Surprisingly, the depth at which this pinch-off takes place does not follow the expected Froude ${ }^{1 / 3}$ power law. Instead, it displays two distinct scaling regimes separated by discrete jumps, both in experiment and in numerical simulations (employing a boundary integral code). We quantitatively explain the above behavior as a capillary wave effect. These waves are created when the top of the cylinder passes the water surface. Our work thus gives further evidence for the nonuniversality of the void collapse.

Many phenomena in fluid dynamics are known to be self-similar [1] and universal, allowing physicists to describe their final outcome without precise knowledge of the initial conditions. Prime examples for such universality are the breakup of an elongated fluid filament inside another viscous fluid [2] and the pinch-off of a liquid droplet in air [3-5]. For the inverse problem [6-10], i.e., when an air bubble pinches off inside a liquid, the dynamics retains a memory of its creation until the very end, indicating nonuniversality. As an example for such a breakup, we examine the air-filled cavity created when a solid object is rapidly submerged through a water surface. The walls of the cavity subsequently collapse due to hydrostatic pressure from the liquid bulk. When the colliding walls meet, a violent jet shoots up into the air. Regardless of the nonuniversality of the pinch-off [7], the location at which it takes place has been reported (experimentally and theoretically) to scale in a continuous fashion with the object velocity for such different systems as spheres on prefluidized sand [11], solid disks [12], spheres and cylinders [13] on water, and even water columns on water [14]. Our experimental and numerical evidence shows the lower limit where this universal scaling is broken through the interference of a second phenomenon unrelated to hydrostatic pressure. Surface waves created as the object passes the water surface significantly alter the pinch-off location in a noncontinuous manner. Similar effects for the breakdown of a universal behavior due to wave interaction have been observed in, e.g., magnetohydrodynamics [15] and turbulence [16].

In our experiment we drag a cylinder with radius $R_{0}=$ $20 \mathrm{~mm}$ and length $l=147 \mathrm{~mm}$ through the surface of a large water tank using a linear motor connected to the cylinder bottom by a rod. We prescribe a constant cylinder velocity $V$ between 0.5 and $2.5 \mathrm{~m} / \mathrm{s}$. With the kinematic viscosity $\nu$ the global Reynolds number $\operatorname{Re}=R_{0} V / \nu$ is of the order of $10^{4}$, while the local Reynolds number $\mathrm{Re}=$ $R \dot{R} / \nu$ defined with the cavity radius $R$ for the point of minimum radius lies between $10^{2}$ and $10^{5}$, demonstrating that inertia dominates viscous effects. Further, with the surface tension coefficient $\sigma, \rho g R_{0} \gg \sigma / R_{0}$; i.e., gravity dominates over surface tension. The relevant dimensionless parameter is thus the Froude number $\mathrm{Fr}=V^{2} /\left(R_{0} g\right)$ with $g=9.81 \mathrm{~m} / \mathrm{s}^{2}$, which in our experiment ranges between 1.2 and 32 .

The shape of the axisymmetric cavity is imaged with a high-speed camera at up to 10,000 frames/ sec, with the vertical coordinate $z$ pointing upwards along the cylinder axis and $r$ being the radial coordinate. Figure 1 shows a typical sequence of the cavity dynamics. We choose the starting position of the lower edge of the cylinder slightly below the water surface to suppress the splash. From there it is pulled downwards with high acceleration such that it has reached its prescribed speed before the top passes the water surface at $t=0$. The submerging cylinder creates an air-filled cavity with an initially cylindrical shape whose side walls immediately start to collapse 1(a). Magnification of the cavity walls as in 1(b) shows that they are not smooth surfaces, but exhibit pronounced ripples. Note that these ripples are of different origin than those observed after pinch-off [17].

From the high-speed video images we extract the closure depth of the cavity $z_{c}$ for cylinder velocities up to $2.5 \mathrm{~m} / \mathrm{s}$. Plotting the closure depth over the Froude number as in Fig. 2, we find two asymptotic regimes. The regime for low Froude numbers obeys a scaling behavior $z_{c} / R_{0} \sim \mathrm{Fr}^{\alpha}$ with an exponent $\alpha=\alpha_{1} \approx 0.1$. This is in contrast to earlier experiments [13], which showed a single continuous scaling behavior with $\alpha \approx \frac{1}{3}$, for which a theoretical explanation in terms of inertia and gravity could be given $[11,13,14]$. At the end of the low Froude regime, the data abruptly depart to an intermediate regime without any experimentally clearly discernible structure. After a pronounced jump we find a third regime, which due to experimental limitations can be observed only between $\mathrm{Fr}=22.4$ and 35.5. While this is too short to ascribe a 

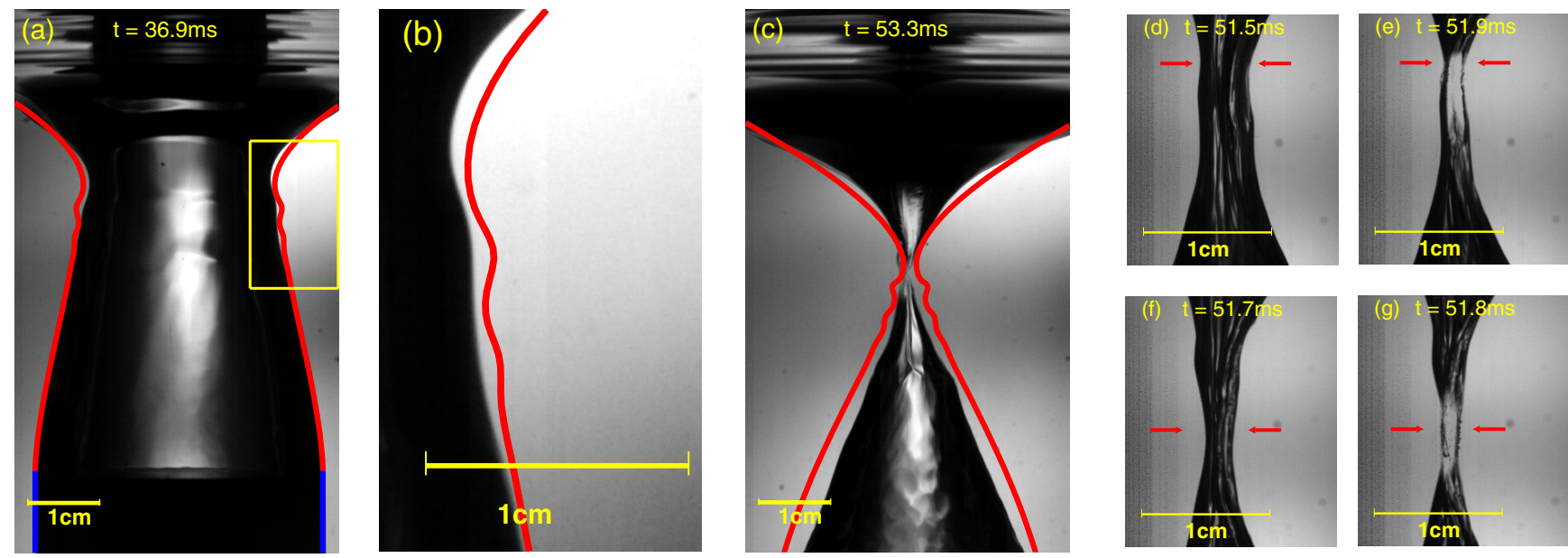

FIG. 1 (color online). (a) - (c) Snapshots of the cavity for $\mathrm{Fr}=11.5$. The area designated by the yellow rectangle in (a) is magnified in (b) illustrating the rippled surface of the cavity walls. Red lines show the numerical simulation. In (a) and (b) video frames are connected to simulation time by matching the cylinder position; for (c) it was more convenient to match the cavity closure times instead. (d) -(g) The transition from one closing ripple to the other. At Fr $=18.8$, (d) and (e), the upper of the two marked ripples closes. At Fr $=20.6$, (f) and (g), it is the lower of the two ripples that pinches.

definite power law behavior, we can nevertheless conclude that this last regime-in contrast to the preceding two regimes - is not disagreeing with the theoretically predicted value of a scaling exponent of $1 / 3$.

To understand the underlying mechanism leading to this discontinuous behavior, we conducted boundary integral simulations. Our numerical results match very well with the high-speed videos without the use of any adjustable parameter; see Fig. 1. The agreement of the wave pattern in Fig. 1(b) can be even further improved when at the expense of introducing a free parameter we allow for a deviation from the precise $90^{\circ}$ angle between the cylinder wall and the free surface, accounting for the free surface being dragged down and resulting in a slightly curved profile close to the cylinder wall.

The numerical simulations allow us to study the ripples in Fig. 1(b) in great detail. As the cylinder top passes the water surface, the rectangular corner between the cylinder wall and the water surface is no longer held in place by the solid boundary of the cylinder. The newly created free surface thus possesses a corner with very high (initially infinite) curvature. Surface tension immediately tries to flatten this surface by pulling the corner diagonally inwards into the fluid bulk. This results in a shock similar to throwing a stone onto a lake, which consequently leads to the formation of capillary waves traveling out- and downward on the free surface. The downward waves can be observed in Fig. 1(b). The shock creates a wave packet containing waves of different frequencies. Each of these waves spreads with a velocity $c=\omega / k$ given by the dispersion relation $\omega^{2}=(\sigma / \rho) k^{3}$, where we assume plane capillary waves (since $k R \gg 1$ during all but the very rapid collapse at the end of the cavity evolution) and $\omega$ designates the angular frequency, $k$ the wave vector, and $\rho$ the density of the liquid.
Using stationary phase approximation, we calculate the dominant wave vector $k^{*}$ at a given distance $x$ from the source at time $t: k^{*}=\left(\frac{2}{3} x / t\right)^{2} \rho / \sigma$. Our simulations allow for an accurate estimate of the dominant wavelength $\lambda$ from the shape of the surface; cf. Fig. 3(a). Comparison with the above equation as in the inset of Fig. 3(a) confirms that the observed ripples on the surface are indeed capillary waves originating from the corner point as the cylinder top passes the water surface. The damping for capillary waves can be estimated as $t_{\mathrm{damp}} \sim 1 /\left(\nu k^{2}\right)$ obtaining, with a wavelength of the order of $6 \mathrm{~mm}$ [see Fig. 1(b)], a damping

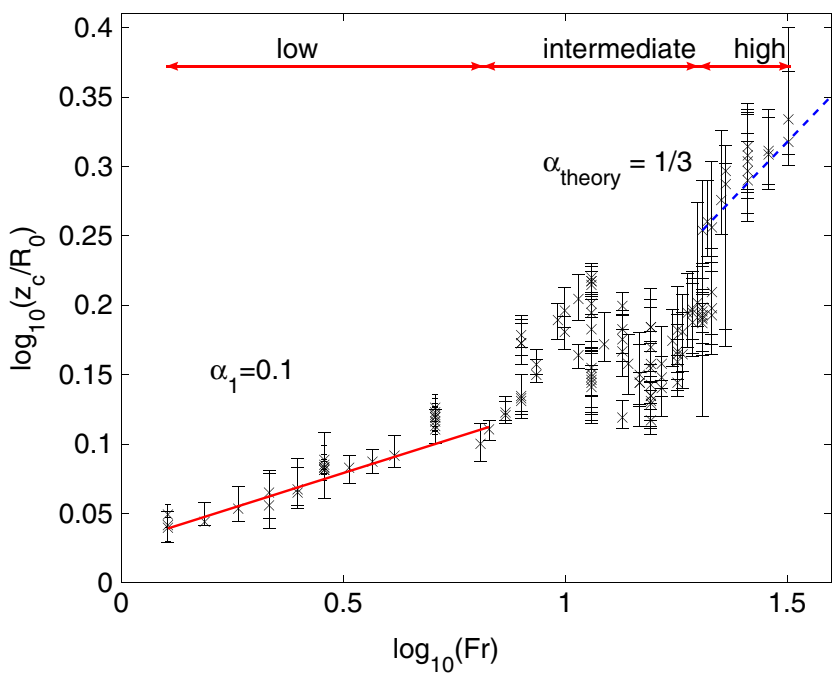

FIG. 2 (color online). The experimental closure depth as a function of the Froude number. The asymptotic regime for low Froude numbers scales with $\alpha_{1} \approx 0.1$ (red solid line). Only in the limit of high Froude numbers does the data not contradict an exponent of $\frac{1}{3}$ (blue dashed line). For the intermediate regime the experiments show no systematic behavior. 


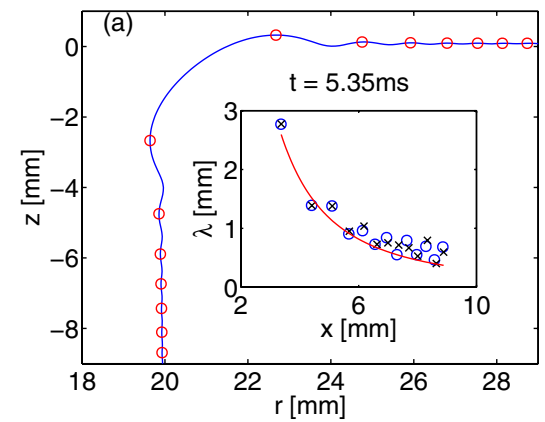

(b)

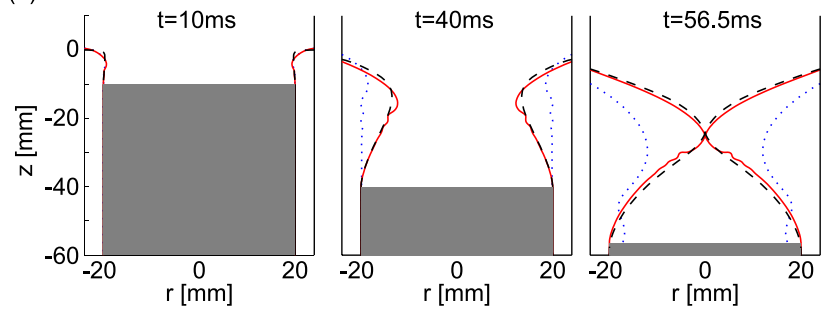

FIG. 3 (color online). (a) Capillary waves for $V=3 \mathrm{~m} / \mathrm{s}$ $(\mathrm{Fr}=45.9)$ as obtained from the simulation. Wave crests are marked by red circles and allow an estimate of the dominant wavelength $\lambda=2 \pi / k$ at a given position. The inset compares these wavelengths for the downward (blue circles) and outward (black crosses) waves to the theoretically expected behavior for capillary waves (red line). (b) The natural cavity dynamics (red solid) as a superposition of two hypothetical settings: without surface tension (black dashed) and without gravity (blue dotted) for $V=1 \mathrm{~m} / \mathrm{s}(\mathrm{Fr}=5.1)$.

time of $900 \mathrm{~ms}$, which is well beyond the closure time of the cavity.

The closing of the cavity is driven by hydrostatic pressure acting on every point of the free surface accelerating it inward as soon as the cylinder has passed. This accelerating force increases with the depth. Thus, points near the top surface start moving early with a small acceleration, while deeper points start with increasing delay, but higher acceleration $[11,13]$. On a rippled surface this process favors the wave crests over the other points. The resulting closure depth will thus be determined by a combination of (i) hydrostatic pressure induced by gravity and (ii) capillary waves created by surface tension.

The numerically obtained closure depth shown in Fig. 4 reasonably reproduces the experimental results. The final regime scales with $\alpha_{2} \approx 0.43$. Considering that the theory from $[11,13,14]$ assumes a purely radial flow pattern and an initially perfectly cylindrical cavity shape, the prediction of $\frac{1}{3}$ is reasonably close to our observed exponent. Our simulations even make the identification of an intermediate regime possible, which due to its small range in Froude numbers cannot be clearly observed in experiments. The shift between numerical and experimental data on the Froude axis can be attributed to the fragility of the measurement process in and around the intermediate regime as well as to small contaminations that lower the surface tension of the water.
The insets in Fig. 4 elucidate precisely how the capillary waves lead to the discontinuous jumps between the different regimes of the closure depth: For Froude numbers near the transition point three different local minima of the radius come very close to meeting their counterpart on the opposite side. In the first regime the uppermost of the three minima closes first and thus determines the closure depth, while in the second regime the one located in the middle is the fastest to reach the central axis. Finally, when the lowest minimum closes before the other two, the third regime is attained. Figures $1(\mathrm{~d})-1(\mathrm{~g})$ show experimental photographs of this effect at the transition point from the intermediate to the high Froude regime.

Since the behavior of the capillary waves is determined by surface tension, one expects that modifications of the surface tension coefficient $\sigma$ should significantly alter the closure depth in the first and any intermediate regimes. For the last regime capillary waves are irrelevant and the scaling behavior of the closure depth can be derived independent of surface tension $[11,13,14]$. We have performed simulations with a tenfold increase and decrease of $\sigma$ as compared to the natural value of $72.8 \mathrm{mN} / \mathrm{m}$ for water as well as for a hypothetical liquid without any surface tension $(\sigma=0)$. As Fig. 5 demonstrates, the Froude number ranges for the three regimes are found to depend indeed strongly on the value of the surface tension coefficient. The length of the first regime significantly enlarges for a higher surface tension coefficient. As expected, the last regime is almost uninfluenced by changes in surface tension. For low surface tension merely the two asymptotic regimes exist and the intermediate regime is not observed anymore. The limiting case completely without surface tension possesses

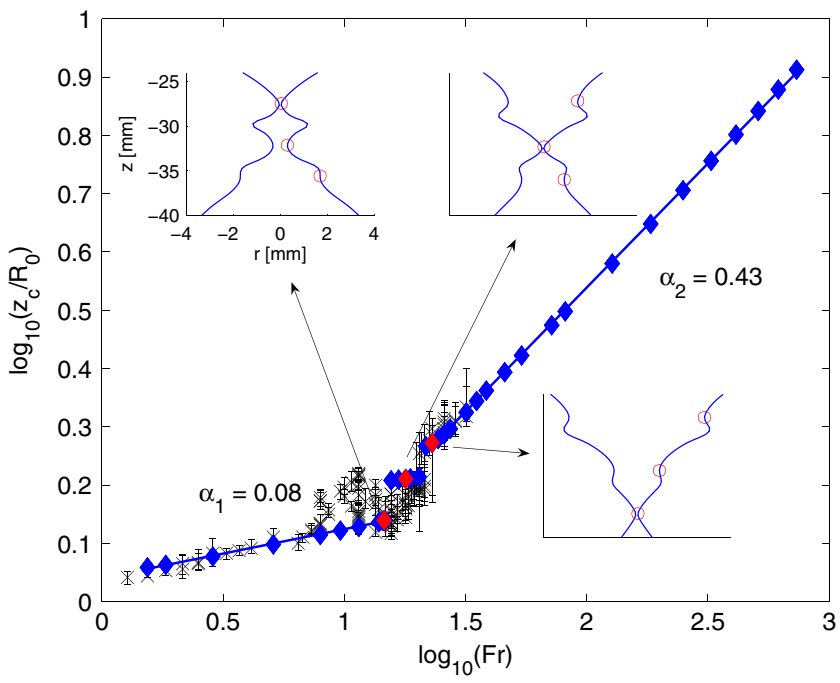

FIG. 4 (color online). Comparison of the experimental closure depth (black crosses) with the numerical data (blue diamonds). The insets illustrate the shape of the cavity at pinch-off for one representative of each regime (axes are the same for all insets). The regimes are determined by which local minimum first meets its counterpart on the central axis. 


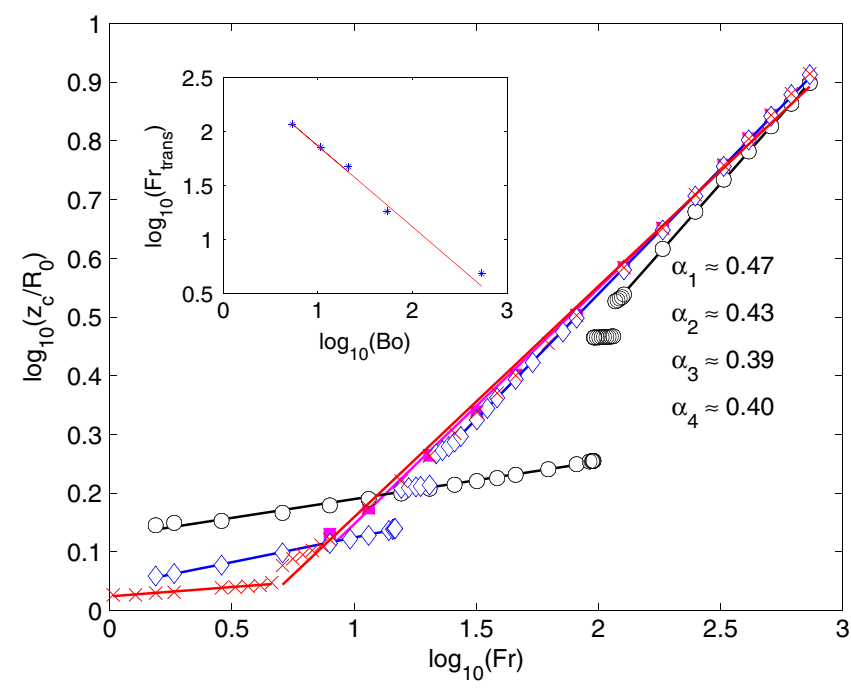

FIG. 5 (color online). The numerical closure depth as a function of the Froude number and corresponding scaling exponents for different surface tension coefficients: $\sigma_{1}=728 \mathrm{mN} / \mathrm{m}$ (black circles), $\sigma_{2}=72.8 \mathrm{mN} / \mathrm{m}$ (blue diamonds), $\sigma_{3}=$ $7.28 \mathrm{mN} / \mathrm{m}$ (red crosses), and $\sigma_{4}=0$ (magenta squares). For $\sigma=0$ no data at low Froude numbers are shown, since axial velocity components during the collapse prohibit the application of the $\mathrm{Fr}^{1 / 3}$ theory. The inset shows the onset of the high Froude number regime as a function of the Bond number with the red line depicting the expected scaling law $\mathrm{Fr}_{\text {trans }} \sim \mathrm{Bo}^{-3 / 4}$.

only one regime in reasonable agreement with the theoretical $\mathrm{Fr}^{1 / 3}$ scaling.

With $x=z_{c}$ and $k^{*} \sim 1 / z_{c}$ we estimate the relevant time scale for the capillary waves as $t_{w} \sim \sqrt{\rho / \sigma} z_{c}^{3 / 2}$ while the time scale for cavity closure is $t_{c} \sim z_{c} / V$. The onset of the high Froude number regime is now readily found by equating the ratio $t_{w} / t_{c}$ to a constant of order 1 . Introducing the Bond number $\mathrm{Bo}=g R_{0}^{2} \rho / \sigma$ and making use of the theoretically expected scaling $z_{c} / R_{0} \sim \mathrm{Fr}^{1 / 3}$, this yields $\mathrm{Fr}_{\text {trans }} \sim \mathrm{Bo}^{-3 / 4}$, which is in good agreement with our numerical observations as shown in the inset of Fig. 5.

The cavity dynamics completely without surface tension and thus deprived of all capillary waves is illustrated by the black dashed lines in Fig. 3(b). The blue dotted lines depict the evolution of a free surface starting with a (nearly) rectangular corner without gravity, allowing us to study the formation of capillary waves in an isolated setting. The cavity dynamics under realistic conditions $\left(g=9.81 \mathrm{~m} / \mathrm{s}^{2}\right.$ and $\sigma=72.8 \mathrm{mN} / \mathrm{m}$ ) is shown in red and can clearly be identified as a superposition of the above-mentioned limiting cases. In the first instants, the real dynamics is almost identical to the one without gravity with its main feature being the capillary waves. Later, hydrostatic pressure becomes more important until finally the cavity approaches the shape of the pure gravity simulation with the capillary waves superposed on the cavity walls.

In conclusion, we have shown that capillary waves created when a submerging object passes the water surface have a strong and lasting influence on the dynamics of the cavity. This influence remains observable until the very end of the cavity collapse. manifesting itself in clearly distinct regimes of the closure depth as a function of the submerging velocity. We have thus illustrated the lower limit to the continuous inertial-gravitational scaling regime observed in [11-14]. Since capillary waves are an unavoidable consequence of disturbances on a water surface, we expect that the effects elaborated in this Letter will be of relevance to a wide range of related phenomena.

We thank L. van Wijngaarden and A. Prosperetti for discussions. This work is part of the program of the Stichting FOM, which is financially supported by NWO.

[1] G. I. Barenblatt, Scaling, Self-similarity, and Intermediate Asymptotics (Cambridge University Press, Cambridge, England, 1996).

[2] M. Tjahjadi, H. A. Stone, and J. M. Ottino, J. Fluid Mech. 243, 297 (1992).

[3] M. P. Brenner, X. D. Shi, and S. R. Nagel, Phys. Rev. Lett. 73, 3391 (1994).

[4] J. Eggers, Rev. Mod. Phys. 69, 865 (1997).

[5] P. Doshi et al., Science 302, 1185 (2003).

[6] J. C. Burton, R. Waldrep, and P. Taborek, Phys. Rev. Lett. 94, 184502 (2005).

[7] R. Bergmann, D. van der Meer, M. Stijnman, M. Sandtke, A. Prosperetti, and D. Lohse, Phys. Rev. Lett. 96, 154505 (2006).

[8] J. M. Gordillo and M. Pérez-Saborid, J. Fluid Mech. 562, 303 (2006).

[9] N.C. Keim, P. Møller, W. W. Zhang, and S. R. Nagel, Phys. Rev. Lett. 97, 144503 (2006).

[10] J. Eggers, M. A. Fontelos, D. Leppinen, and J. H. Snoeijer, Phys. Rev. Lett. 98, 094502 (2007).

[11] D. Lohse, R. Bergmann, R. Mikkelsen, C. Zeilstra, D. van der Meer, M. Versluis, K. van der Weele, M. van der Hoef, and H. Kuipers, Phys. Rev. Lett. 93, 198003 (2004).

[12] J. W. Glasheen and T. A. McMahon, Phys. Fluids 8, 2078 (1996).

[13] V. Duclaux, F. Caillé, C. Duez, C. Ybert, L. Bocquet, and C. Clanet, J. Fluid Mech. 591, 1 (2007).

[14] H. N. Oguz, A. Prosperetti, and A. R. Kolaini, J. Fluid Mech. 294, 181 (1995).

[15] A. Basu and J. K. Bhattacharjee, J. Stat. Mech. (2005) $\mathrm{P} 07002$

[16] F. Cioffi, F. Gallerano, and G. Troiani, J. Hydraul. Res. 44, 155 (2006).

[17] T. Grumstrup, J. B. Keller, and A. Belmonte, Phys. Rev. Lett. 99, 114502 (2007). 